\title{
A theoretical calculation of the cosmological constant based on the theory of vacuum mechanics
}

\author{
Xiao-Song Wang \\ Institute of Mechanical and Power Engineering, Henan Polytechnic University, Jiaozuo, Henan Province, 454000, China
}

(Dated: December 21, 2019)

\begin{abstract}
Lord Kelvin believes that the electromagnetic aether must also generate gravity. Based on a theorem of V. Fock on the mass tensor of an elastic continuum, the contravariant energy-momentum tensor of the $\Omega(1)$ substratum is established. Quasi-static solutions of the gravitational field equations in vacuum are obtained. Based on an assumption, relationships between the contravariant energy-momentum tensor of the $\Omega(1)$ substratum and the contravariant metric tensor are obtained. Thus, the cosmological constant is derived theoretically. The $\Omega(1)$ substratum, or we say the electromagnetic aether, may be a possible candidate of the dark energy. The zero-point energy of electromagnetic fields will not appear as a source term in the generalized Einstein's equations. Some people believed that all kinds of energies should appear as source terms in the Einstein's equations. It may be this belief that leads to the so called cosmological constant problem. The mass density of the electromagnetic aether is equivalent to that 31.33195 protons contained in a box with a volume of $1.0 \mathrm{~m}^{3}$.
\end{abstract}

Keywords: cosmological constant; dark energy; general relativity; vacuum mechanics; electromagnetic aether.

\section{INTRODUCTION}

It is known that the cosmological constant problem is one of the puzzles in physics today $[1,2]$. Theoretical interpretation of the small value of the cosmological constant is still open [3].

In 1917, A. Einstein thought that his equations of gravitational fields should be revised to be ([4], p. 410)

$$
R_{\mu \nu}-\frac{1}{2} g_{\mu \nu} R+\Lambda g_{\mu \nu}=-\kappa T_{\mu \nu}^{m}
$$

where $g_{\mu \nu}$ is the metric tensor of a Riemannian spacetime, $R_{\mu \nu}$ is the Ricci tensor, $R \equiv g^{\mu \nu} R_{\mu \nu}$ is the scalar curvature, $g^{\mu \nu}$ is the contravariant metric tensor, $\kappa$ is Einstein's gravitational constant, $c$ is the speed of light in vacuum, $T_{\mu \nu}^{m}$ is the energy-momentum tensor of a matter system, $\Lambda$ is the cosmological constant.

However, it seems that the cosmological constant $\Lambda$ is unnecessary when Hubble discovered the expansion of the universe. Thus, Einstein abandoned the term $\Lambda g_{\mu \nu}$ in Eqs. (1) and returned to his original equations ([4], p. 410).

The value of the cosmological constant $\Lambda$ is also related to the energy-momentum tensor of vacuum ([4], p. 411). The existences and characters of dark matter and dark energy are still controversy, refers to, for instance, [5-9].

The purpose of this manuscript is to propose an interpretation of the cosmological constant and a model of dark energy based on the theory of vacuum mechanics [10-13].

\section{POSSIBLE ORIGIN OF THE COSMOLOGICAL CONSTANT}

Lord Kelvin once said [14]:" That there must be a medium forming a continuous material communication throughout space to the remotest visible body is a fundamental assumption in the undulatory theory of light." Lord Kelvin believes that the electromagnetic aether must also generate gravity [14, 15]. Following Lord Kelvin, we speculate that the particles which constitute the electromagnetic aether will gravitate with each other and matter.

Recently $[13,16]$, we propose an assumption that the particles that constitute the $\Omega(1)$ substratum, or the electromagnetic aether, are sinks in the $\Omega(0)$ substratum. Thus, the electromagnetic aether will also generate gravity [11]. Further, generalized Einstein's equations in a special class of non-inertial reference frames are derived based on the theory of vacuum mechanics [16]. If the non-inertial reference frame is quasi-inertial and the gravitational field is weak, then the generalized Einstein's equations reduce to $[13,16]$

$$
R_{\mu \nu}-\frac{1}{2} g_{\mu \nu} R=\frac{\kappa}{g_{0}}\left(T_{\mu \nu}^{m}+T_{\mu \nu}^{\Omega(1)}\right),
$$

where $T_{\mu \nu}^{m}$ and $T_{\mu \nu}^{\Omega(1)}$ are the energy-momentum tensors of matter system and the $\Omega(1)$ substratum respectively, $g_{0}=\operatorname{Det} g_{\mu \nu}$.

Comparing Eqs.(2) and Eqs.(1), it is natural for us to speculate that the cosmological term $\Lambda g_{\mu \nu}$ in Eqs.(1) may stem from the term $\kappa / g_{0} T_{\mu \nu}^{\Omega(1)}$ in Eqs.(2). We will investigate this clue in the following sections.

\section{GRAVITATIONAL FIELD EQUATIONS IN VACUUM}

It is known that the movements of particles are described by special relativistic mechanics [17-19]. Thus, the movements of continuously distributed matters and fields should also be studied based on special relativity $[17,19]$. It is known that Maxwell's equations are valid in 
the frame of reference that attached to the $\Omega(1)$ substratum [10]. In order to compare our theory with Newtonian theory of gravitation, which is non-relativistic, it is better to single out the speed of light $c$ as a large parameter. Therefore, we introduce the following notations

$$
x^{0}=t, x^{1}=x, x^{2}=y, x^{3}=z,
$$

where $\{o, x, y, z\}$ is a Cartesian coordinate system for a three-dimensional Euclidean space that attached to the $\Omega(1)$ substratum, $\{o, t\}$ is a one-dimensional time coordinate.

We denote this reference frame as $S_{\Omega(1)}$. An inertial reference frame is defined to be a coordinates system which is static or moving with a constant velocity relative to the reference frame $S_{\Omega(1)}$ [13]. We will use Greek indices $\alpha, \beta, \mu, \nu$, etc., for the range $0,1,2,3$ and use Latin indices $i, j, k$, etc., for the range $1,2,3$. According to a theorem of V. Fock, the characteristics of the generalized wave equation, or d'Alembert equation

$$
\square_{\gamma}^{2} \stackrel{\text { def }}{=} \frac{1}{\sqrt{-\gamma}} \frac{\partial}{\partial x^{\alpha}}\left(\sqrt{-\gamma} \gamma^{\alpha \beta} \frac{\partial \psi}{\partial x^{\beta}}\right)=0
$$

is $([20]$, p. 432$)$

$$
\gamma^{\mu \nu} \frac{\partial \omega}{\partial x^{\mu}} \frac{\partial \omega}{\partial x^{\nu}}=0
$$

where $\psi$ is a field variable, $\gamma_{\mu \nu}$ is a metric tensor, $\gamma=$ Det $\gamma_{\mu \nu}, \omega\left(x^{0}, x^{1}, x^{2}, x^{3}\right)$ is the characteristics.

In 2008 [10], we suppose that vacuum is filled with a kind of continuously distributed material which may be called the $\Omega(1)$ substratum or the electromagnetic aether. Maxwell's equations in vacuum are derived by methods of continuum mechanics based on this mechanical model of vacuum and a source and sink flow model of electric charges [10]. Based on the Maxwell's equations, the law of propagation of an electromagnetic wave front in this reference frame $S_{\Omega(1)}$ can be derived and can be written as $([20], \mathrm{p} 13)$

$$
\frac{1}{c^{2}}\left(\frac{\partial \omega}{\partial x^{0}}\right)^{2}-\left(\frac{\partial \omega}{\partial x^{1}}\right)^{2}-\left(\frac{\partial \omega}{\partial x^{2}}\right)^{2}-\left(\frac{\partial \omega}{\partial x^{3}}\right)^{2}=0
$$

where $\omega\left(x^{0}, x^{1}, x^{2}, x^{3}\right)$ is an electromagnetic wave front, $c$ is the velocity of light in the reference frame $S_{\Omega(1)}$.

Since there is no atoms in vacuum, the gravitational field is weak. Thus, $g_{0} \approx-1$ and the field equations (2) reduce to

$$
R^{\mu \nu}-\frac{1}{2} g^{\mu \nu} R=-\kappa T_{\Omega(1)}^{\mu \nu} .
$$

Comparing Eq. (6) and Eq. (5), the contravariant metric tensor $g^{\mu \nu}$ must have the following limit values at infinity ([20], p. 196)

$$
\left(g^{00}\right)_{\infty}=\frac{1}{c^{2}} ;\left(g^{0 i}\right)_{\infty}=0 ;\left(g^{i k}\right)_{\infty}=-\delta_{i k},
$$

where $\delta_{i k}$ is the Kronecker symbol.

Eqs. (8) can be written as

$$
\left(g^{\mu \nu}\right)_{\infty}=\left(\begin{array}{rrrr}
\frac{1}{c^{2}} & 0 & 0 & 0 \\
0 & -1 & 0 & 0 \\
0 & 0 & -1 & 0 \\
0 & 0 & 0 & -1
\end{array}\right)
$$

The corresponding limit values of the metric tensor $g_{\mu \nu}$ will be ([20], p. 196)

$$
\left(g_{00}\right)_{\infty}=c^{2} ;\left(g_{0 i}\right)_{\infty}=0 ;\left(g_{i k}\right)_{\infty}=-\delta_{i k} .
$$

Eqs. (10) can be written as

$$
\left(g_{\mu \nu}\right)_{\infty}=\left(\begin{array}{rrrr}
c^{2} & 0 & 0 & 0 \\
0 & -1 & 0 & 0 \\
0 & 0 & -1 & 0 \\
0 & 0 & 0 & -1
\end{array}\right) .
$$

\section{ENERGY-MOMENTUM TENSOR OF THE $\Omega(1)$ SUBSTRATUM}

It is known that Maxwell's equations are valid in the frame of reference $S_{\Omega(1)}$ [10]. From the view point of a microscopic observer, the $\Omega(1)$ substratum behaves as an elastic continuum [10]. Thus, according to a theorem of $\mathrm{V}$. Fock on the mass tensor of an elastic continuum, the contravariant mass tensor $M_{S}^{\mu \nu}$ of the $\Omega(1)$ substratum for the coordinate system $S \triangleq\{c t, x, y, z\}$ can be written as ([20], p. 106)

$$
\begin{aligned}
& M_{S}^{00}=\rho_{1}+\frac{1}{c^{2}}\left(\frac{1}{2} \rho_{1} v^{2}+\rho_{1} \Pi\right) \\
& M_{S}^{0 i}=\frac{\rho_{1} v_{i}}{c}+\frac{v_{i}\left(\frac{\rho_{1} v^{2}}{2}+\rho_{1} \Pi\right)-\sum_{k} p_{i k} v_{k}}{c^{3}} \\
& M_{S}^{i j}=\frac{\rho_{1} v_{i} v_{j}-p_{i j}}{c^{2}}
\end{aligned}
$$

where $\rho_{1}$ is the rest mass density of a volume element of the $\Omega(1)$ substratum in the laboratory frame relative to which the volume element moves with velocity $\mathbf{v}, v=\sqrt{\mathbf{v} \cdot \mathbf{v}}, p_{i j}$ is the static stress tensor of the $\Omega(1)$ substratum resulted from the potentials between the particles,

$$
\begin{gathered}
\Pi=-\frac{p}{\rho_{1}^{*}}+\int_{0}^{p} \frac{1}{\rho_{1}^{*}} d p, \\
\rho_{1}^{*}=\rho_{1} \sqrt{1-\frac{v^{2}}{c^{2}}},
\end{gathered}
$$

$p \delta_{i j}=\left\langle p_{i j}\right\rangle,\langle\rangle$ denotes the averaged macroscopic value in the sense of statistical physics, $\delta_{i j}$ is the Kronecker symbol.

The relationships between the contravariant mass tensor $M_{S}^{\mu \nu}$ in the coordinate system $\{c t, x, y, z\}$ and the 
contravariant mass tensor $M_{\Omega(1)}^{\mu \nu}$ in the present coordinate system $\{t, x, y, z\}$ are ([20], p. 198)

$$
\begin{aligned}
& M_{\Omega(1)}^{00}=\frac{1}{c^{2}} M_{S}^{00}, \\
& M_{\Omega(1)}^{0 i}=\frac{1}{c} M_{S}^{0 i}, \\
& M_{\Omega(1)}^{i j}=M_{S}^{i j} .
\end{aligned}
$$

Thus, the contravariant mass tensor $M_{\Omega(1)}^{\mu \nu}$ of the $\Omega(1)$ substratum in the coordinate system $\{c t, x, y, z\}$ can be written as

$$
\begin{aligned}
& M_{\Omega(1)}^{00}=\frac{\rho_{1}}{c^{2}}+\frac{1}{c^{4}}\left(\frac{1}{2} \rho_{1} v^{2}+\rho_{1} \Pi\right) \\
& M_{\Omega(1)}^{0 i}=\frac{\rho_{1} v_{i}}{c^{2}}+\frac{v_{i}\left(\frac{\rho_{1} v^{2}}{2}+\rho_{1} \Pi\right)-\sum_{k} p_{i k} v_{k}}{c^{4}} \\
& M_{\Omega(1)}^{i j}=\frac{\rho_{1} v_{i} v_{j}-p_{i j}}{c^{2}}
\end{aligned}
$$

Then, for the present coordinate system $\{t, x, y, z\}$, the contravariant energy-momentum tensor $T_{\Omega(1)}^{\mu \nu}=M^{\mu \nu} c^{2}$ can be written as

$$
\begin{aligned}
& T_{\Omega(1)}^{00}=\rho_{1}+\frac{1}{c^{2}}\left(\frac{1}{2} \rho_{1} v^{2}+\rho_{1} \Pi\right) \\
& T_{\Omega(1)}^{0 i}=\rho_{1} v_{i}+\frac{v_{i}\left(\frac{\rho_{1} v^{2}}{2}+\rho_{1} \Pi\right)-\sum_{k} p_{i k} v_{k}}{c^{2}} \\
& T_{\Omega(1)}^{i j}=\rho_{1} v_{i} v_{j}-p_{i j} .
\end{aligned}
$$

Assumption 1 We have the following relationships

$$
\left\langle\rho_{1} v_{i} v_{j}-p_{i j}\right\rangle=\alpha_{0} \rho_{1 \mathrm{e}} \delta_{i j},
$$

where \langle\rangle denotes the averaged macroscopic value in the sense of statistical physics from the view point of the macroscopic observer, $\delta_{i j}$ is the Kronecker symbol, $\rho_{1 \mathrm{e}}$ is the energy density of a volume element of the $\Omega(1)$ substratum, $\alpha_{0}$ is a parameter to be determined.

Einstein's mass energy formula for a particle can be written as

$$
E=m c^{2}
$$

where $E$ is the energy of the particle, $m$ is the mass of the particle, $c$ is the velocity of light in vacuum.

Applying Einstein's formula (27) to a volume element of the $\Omega(1)$ substratum, we have

$$
\rho_{1 \mathrm{e}}=\frac{m c^{2}}{V}=\frac{m_{r} c^{2}}{V \sqrt{1-\frac{v^{2}}{c^{2}}}}=\frac{\rho_{1} c^{2}}{\sqrt{1-\frac{v^{2}}{c^{2}}}}
$$

where $V$ is the volume of a volume element, $m_{r}$ is the rest mass of a volume element, $v$ is the velocity of the volume element.
From Eq.(25), Eq.(26) and Eq.(28), we have

$$
\left\langle T_{\Omega(1)}^{i j}\right\rangle=\frac{\alpha_{0} \rho_{1} c^{2}}{\sqrt{1-\frac{v^{2}}{c^{2}}}} \delta_{i j}, i, j=1,2,3 .
$$

We suppose that $v^{2} \ll c^{2}$. Thus, $v^{2} / c^{2} \approx 0$. Neglecting those terms of order of $1 / c^{2}$ in Eq.(23), Eqs.(24) and Eqs.(29) and replacing $T_{\Omega(1)}^{i j}$ by $\left\langle T_{\Omega(1)}^{i j}\right\rangle$ in Eqs.(29), a macroscopic observer will obtain

$$
\begin{aligned}
& T_{\Omega(1)}^{00} \approx \rho_{1}, \\
& T_{\Omega(1)}^{0 i} \approx \rho_{1} v_{i}, \\
& T_{\Omega(1)}^{i j} \approx \alpha_{0} \rho_{1} c^{2} \delta_{i j} .
\end{aligned}
$$

Using Eq.(30-32) and Eqs.(10), we have

$$
T_{\Omega(1)} \stackrel{\text { def }}{=} g_{\alpha \beta} T_{\Omega(1)}^{\alpha \beta} \approx\left(1-3 \alpha_{0}\right) \rho_{1} c^{2}
$$

\section{QUASI-STATIC SOLUTIONS OF THE GRAVITATIONAL FIELD EQUATIONS IN VACUUM}

The field equations (7) can be written as ([20], p. 198)

$$
R^{\mu \nu}=-\kappa\left(T_{\Omega(1)}^{\mu \nu}-\frac{1}{2} g^{\mu \nu} T_{\Omega(1)}\right) .
$$

Using Eqs.(8) and Eq.(30-33), we have

$$
\begin{aligned}
& T_{\Omega(1)}^{00}-\frac{1}{2} g^{00} T_{\Omega(1)} \approx \frac{\left(1+3 \alpha_{0}\right) \rho_{1}}{2}, \\
& T_{\Omega(1)}^{0 i}-\frac{1}{2} g^{0 i} T_{\Omega(1)} \approx \rho_{1} v_{i}, \\
& T_{\Omega(1)}^{i j}-\frac{1}{2} g^{i j} T_{\Omega(1)} \approx \frac{\left(1-\alpha_{0}\right) \rho_{1} c^{2}}{2} \delta_{i j} .
\end{aligned}
$$

Inertial reference frames are harmonic. For harmonic coordinates, we have approximately ([20], p. 198)

$$
R^{\mu \nu}=-\frac{1}{2 c^{2}} \frac{\partial^{2} g^{\mu \nu}}{\partial\left(x^{0}\right)^{2}}+\frac{1}{2} \nabla^{2} g^{\mu \nu},
$$

where

$$
\nabla^{2} g^{\mu \nu}=\frac{\partial^{2} g^{\mu \nu}}{\partial\left(x^{1}\right)^{2}}+\frac{\partial^{2} g^{\mu \nu}}{\partial\left(x^{2}\right)^{2}}+\frac{\partial^{2} g^{\mu \nu}}{\partial\left(x^{3}\right)^{2}} .
$$

Since we are interested in the quasi-static behavior of $g^{\mu \nu}$, we assume that

$$
\frac{\partial^{2} g^{\mu \nu}}{c^{2} \partial\left(x^{0}\right)^{2}}=0
$$

Putting Eq.(38) and Eq.(35-37) into Eq.(34) and using Eqs.(40), we obtain

$$
\begin{aligned}
& \nabla^{2} g^{00} \approx-\left(1+3 \alpha_{0}\right) \kappa \rho_{1}, \\
& \nabla^{2} g^{0 i} \approx-2 \kappa \rho_{1} v_{i}, \\
& \nabla^{2} g^{i j} \approx-\left(1-\alpha_{0}\right) \kappa \rho_{1} c^{2} \delta_{i j} .
\end{aligned}
$$


Newton's gravitational potential $U$ satisfies the following equation ([20], p. 199)

$$
\nabla^{2} U=4 \pi G_{n} \rho_{1}
$$

where $G_{n}$ is Newton's gravitational constant.

Following V. Fock ([20], p199), we introduce the following gravitational vector potentials $U_{i}$ which satisfy

$$
\nabla^{2} U_{i}=4 \pi G_{n} \rho_{1} v_{i}
$$

Proposition 2 Suppose that the following conditions are valid: (1) $U \approx 0$ and $U_{i} \approx 0$ at infinity; (2) in the spherical coordinates $(r, \theta, \psi), U$ and $g^{\mu \nu}$ are axially symmetric, i.e., they do not vary with the azimuthal angle $\psi$; (3) the contravariant metric tensor $g^{\mu \nu}$ depends on $r$ and $\theta$ as the Newton potential $U$; (4) the law of propagation of an electromagnetic wave front, i.e., Eq.(6), is valid in the reference frame $S_{\Omega(1)}$ at infinity. Then, the solutions of Eq.(41-43) are

$$
\begin{aligned}
g^{00} & \approx \frac{1}{c^{2}}-\frac{\left(2+6 \alpha_{0}\right) U}{c^{4}}, \\
g^{0 i} & \approx-\frac{4 U_{i}}{c^{4}} \\
g^{i j} & \approx\left[-1+\frac{\left(2-2 \alpha_{0}\right) U}{c^{2}}\right] \delta_{i j} .
\end{aligned}
$$

Proof of Proposition 2. From Eq.(44), we have

$$
\rho_{1}=\frac{1}{4 \pi G_{n}} \nabla^{2} U
$$

Putting Eq.(49) into Eq.(41), we have

$$
\nabla^{2}\left(g^{00}+\beta_{0} U\right) \approx 0,
$$

where

$$
\beta_{0}=\frac{\left(1+3 \alpha_{0}\right) \kappa}{4 \pi G_{n}}
$$

The Einstein's gravitational constant $\kappa$ is ([17], p. 429)

$$
\kappa=\frac{8 \pi G_{n}}{c^{4}}
$$

Thus, the parameter $\beta_{0}$ can be written as

$$
\beta_{0}=\frac{2+6 \alpha_{0}}{c^{4}} .
$$

Eq.(50) is a Laplace's equation. Since $U$ and $g^{00}$ are axially symmetric, a general form of solution of Eq.(50) can be obtained by using the spherical coordinates $(r, \theta, \varphi)([21], \mathrm{p} 132)$

$$
g^{00}(r, \theta)+\beta_{0} U(r, \theta) \approx \sum_{n}\left(A_{n} r^{n}+\frac{B_{n}}{r^{n+1}}\right) P_{n}(\cos \theta)
$$

where $A_{n}$ and $B_{n}$ are arbitrary constants, $n$ are integers, $P_{n}(x)$ are Legendre's functions of the first kind which is defined by

$$
P_{n}(x)=\frac{1}{2^{n} n !} \frac{\mathrm{d}^{n}}{\mathrm{~d} x^{n}}\left(x^{2}-1\right)^{n} .
$$

We have assumed that the contravariant metric tensor $g^{\mu \nu}$ will depend on $r$ and $\theta$ as the Newton potential $U$. Thus, we set $n=0$ and $B_{n}=0$ in Eq.(54) and obtain

$$
g^{00}+\beta_{0} U \approx A_{0},
$$

where $A_{0}$ is a constant to be determined.

Similarly, we have

$$
\begin{aligned}
& g^{0 i}+\beta_{1} U_{i} \approx A_{1}, \\
& g^{i j}+\beta_{2} U \delta_{i j} \approx A_{2} \delta_{i j},
\end{aligned}
$$

where $A_{1}$ and $A_{2}$ are constants to be determined,

$$
\begin{aligned}
& \beta_{1}=\frac{4}{c^{4}}, \\
& \beta_{2}=\frac{2-2 \alpha_{0}}{c^{2}} .
\end{aligned}
$$

Noticing $U \approx U_{i} \approx 0$ at infinity and using Eqs.(8), we have

$$
A_{0}=\frac{1}{c^{2}}, A_{1}=0, A_{2}=-1
$$

Thus, the solutions of Eqs.(41-43) are Eqs.(46-46).

Now the variant metric tensor $g_{\mu \nu}$ can be derived from the contravariant metric tensor $g^{\mu \nu}$ and the results are ([20], p. 201).

$$
\begin{aligned}
& g_{00} \approx c^{2}-\left(2+6 \alpha_{0}\right) U, \\
& g_{0 i} \approx-\frac{4 U_{i}}{c^{4}}, \\
& g_{i j} \approx\left[-1-\frac{\left(2-2 \alpha_{0}\right) U}{c^{2}}\right] \delta_{i j} .
\end{aligned}
$$

\section{A THEORETICAL DERIVATION OF THE COSMOLOGICAL CONSTANT}

We speculate that the $\Omega(1)$ substratum may be responsible for the so called dark energy. Following this idea, we set out to explore the possible value of the parameter $\alpha_{0}$ in Eqs.(26).

We have supposed that the velocity $v$ of the volume element of the $\Omega(1)$ substratum is small, i.e., $v^{2} \ll c^{2}$. Thus, the terms $\rho_{1} v_{i}$ in Eqs.(31) may be neglected. Then according to Eqs.(30-32), the contravariant energymomentum tensor $T_{\Omega(1)}^{\mu \nu}$ of the $\Omega(1)$ substratum can be written as

$$
T_{\Omega(1)}^{\mu \nu} \approx \rho_{1} c^{2}\left(\begin{array}{rrrr}
\frac{1}{c^{2}} & 0 & 0 & 0 \\
0 & \alpha_{0} & 0 & 0 \\
0 & 0 & \alpha_{0} & 0 \\
0 & 0 & 0 & \alpha_{0}
\end{array}\right) .
$$


Since $v$ and $\rho_{1}$ are small enough, Newton's gravitational potential $U$ in Eq.(44) and the gravitational vector potentials $U_{i}$ in Eqs.(45) are small relative to $c^{2}$. Thus, the terms involving $U$ or $U_{i}$ in Eq.(46-48) may be neglected. Then, the contravariant metric tensor $g^{\mu \nu}$ of the $\Omega(1)$ substratum can be written as

$$
g^{\mu \nu} \approx\left(\begin{array}{rrrr}
\frac{1}{c^{2}} & 0 & 0 & 0 \\
0 & -1 & 0 & 0 \\
0 & 0 & -1 & 0 \\
0 & 0 & 0 & -1
\end{array}\right)
$$

In section II we speculate that the cosmological term $\Lambda g_{\mu \nu}$ in Eqs.(1) may stem from the term $\kappa / g_{0} T_{\mu \nu}^{\Omega(1)}$ in Eqs.(2). Now comparing Eq.(65) and Eq.(66), it is natural for us to speculate that $g^{\mu \nu}$ may depends on $T_{\Omega(1)}^{\mu \nu}$ approximately linearly. Therefore, we introduce the following assumption.

\section{Assumption $3 \alpha_{0}=-1$.}

Now comparing Eq.(65) and Eq.(66) and applying Assumption 3, we obtain the following relationships

$$
T_{\mu \nu}^{\Omega(1)} \approx \rho_{1} c^{2} g_{\mu \nu} .
$$

Now we study the gravitational field of a system of matter and the $\Omega(1)$ substratum. We suppose that the gravitational field is weak, i.e., Eq.(66) is valid. Thus, Eqs.(67) are still valid. Putting Eqs.(67) into Eqs.(2) and assuming $g_{0} \approx-1$, we have

$$
R_{\mu \nu}-\frac{1}{2} g_{\mu \nu} R \approx-\kappa T_{\mu \nu}^{m}-\kappa \rho_{1} c^{2} g_{\mu \nu}
$$

Comparing Eqs.(68) and Eqs.(1), we obtain the following relationship

$$
\Lambda=\kappa \rho_{1} c^{2}
$$

Using Eq.(52), Eq.(69) can be written as

$$
\Lambda=\frac{8 \pi G_{n} \rho_{1}}{c^{2}}
$$

\section{A THEORETICAL CALCULATION OF THE MASS DENSITY OF THE $\Omega(1)$ SUBSTRATUM}

It is known that the mass density of the electromagnetic aether is an open question since eighteenth century [22-24]. Now we may have a chance to discuss this problem.

From Eq.(70), we have

$$
\rho_{1}=\frac{\Lambda c^{2}}{8 \pi G_{n}} .
$$

Thus, the energy density $\rho_{1 \mathrm{e}}$ of the $\Omega(1)$ substratum is

$$
\rho_{1 \mathrm{e}}=\rho_{1} c^{2}=\frac{\Lambda c^{4}}{8 \pi G_{n}} .
$$

We have the following data [25-27]

$$
\begin{aligned}
\Lambda & =1.088(30) \times 10^{-52} \mathrm{~m}^{-2}, \\
c & =2.99792458 \times 10^{8} \mathrm{~m} \cdot \mathrm{s}^{-1}, \\
G_{n} & =6.67430(15) \times 10^{-11} \mathrm{~m}^{-2} \mathrm{~kg}^{-1} \mathrm{~s}^{-2} .
\end{aligned}
$$

Using these data, we obtain

$$
\begin{aligned}
\rho_{1} & =5.24065 \times 10^{-26} \mathrm{~kg} \cdot \mathrm{m}^{-3}, \\
\rho_{1 \mathrm{e}} & =4.41006 \times 10^{-9} \mathrm{~J} \cdot \mathrm{m}^{-3} .
\end{aligned}
$$

The mass $m_{\mathrm{p}}$ of a proton is [25-27]

$$
m_{\mathrm{p}}=1.67262192369(51) \times 10^{-27} \mathrm{~kg} \text {. }
$$

Comparing Eq.(78) and Eq.(76), the mass density of the $\Omega(1)$ substratum is equivalent to that 31.33195 protons contained in a box with a volume of $1.0 \mathrm{~m}^{3}$.

\section{A POSSIBLE CANDIDATE OF THE DARK ENERGY}

According to Assumption 3 and Eq.(67), the origin of the cosmological term $\Lambda g_{\mu \nu}$ in Eqs. (1) is the energymomentum tensors $T_{\mu \nu}^{\Omega(1)}$ of the $\Omega(1)$ substratum. Therefore, the $\Omega(1)$ substratum, or we say the electromagnetic aether, may be a candidate of the so called concept of dark energy [2].

Applying Assumption 3 and Eq.(28), Eqs.(26) can be written as

$$
\left\langle\rho_{1} v_{i} v_{j}-p_{i j}\right\rangle=-\frac{\rho_{1} c^{2}}{\sqrt{1-\frac{v^{2}}{c^{2}}}} \delta_{i j} .
$$

The physical meaning of $\left\langle\rho_{1} v_{i} v_{j}\right\rangle$ is the macroscopic dynamical pressure in the $\Omega(1)$ substratum. $\left\langle-p_{i j}\right\rangle$ is the macroscopic static pressure in the $\Omega(1)$ substratum. Thus, the physical meaning of $\left\langle\rho_{1} v_{i} v_{j}-p_{i j}\right\rangle$ is the macroscopic total pressure in the $\Omega(1)$ substratum. Eqs.(79) show that the macroscopic total pressure in the $\Omega(1)$ substratum is negative. This negativeness of the pressure of the $\Omega(1)$ substratum coincides with the negativeness of the pressure of dark energy [2, 28]. Thus, the $\Omega(1)$ substratum may be a possible candidate of the dark energy [2].

\section{AN OPINION ON THE COSMOLOGICAL CONSTANT PROBLEM}

Various solutions have been proposed to solve the cosmological constant problem [28]. The first class of solutions is to modify the theory of gravitation. The second class of solutions is to revise the standard model of particle physics.

According to the new theory of gravitation $[11,13,16]$, two sinks in the $\Omega(0)$ substratum will gravitate with each 
other. The particles that constitute the $\Omega(1)$ substratum are sinks in the $\Omega(0)$ substratum. Thus, particles of the $\Omega(1)$ substratum will also generate gravity [11]. Therefore, only two kinds of energy-momentum tensor, i.e., $T_{\mu \nu}^{m}$ and $T_{\mu \nu}^{\Omega(1)}$, will appear as source terms in the generalized Einstein's equations (2). Not all kinds of energies will appear as source terms in the generalized Einstein's equations (2). Thus, the zero-point energy of electromagnetic fields will not appear as a source term in the generalized Einstein's equations (2).

From Eq.(67), we notice that the origin of the cosmological term $\Lambda g_{\mu \nu}$ in Eqs. (1) is the energy-momentum tensors $T_{\mu \nu}^{\Omega(1)}$ of the $\Omega(1)$ substratum. Thus, the cosmological term $\Lambda g_{\mu \nu}$ may not result from the zero-point energy of electromagnetic fields.

However, some people believed that all kinds of energies should appear as source terms in the Einstein's equations $[2,28]$. It may be this belief that leads to the so called cosmological constant problem.

\section{CONCLUSION}

The cosmological constant problem is one of the puzzles in physics. Lord Kelvin believes that the electromagnetic aether must also gravitate with each other and matter. According to a theorem of V. Fock on the mass tensor of an elastic continuum, the contravariant energymomentum tensor of the $\Omega(1)$ substratum is established. Quasi-static solutions of the gravitational field equations in vacuum are obtained. Based on an assumption, relationships between the contravariant energy-momentum tensor of the $\Omega(1)$ substratum and the contravariant metric tensor are obtained. Thus, the cosmological constan$\mathrm{t}$ is derived theoretically. Therefore, the $\Omega(1)$ substratum may be a possible candidate of the dark energy. As a byproduct, the mass density of the $\Omega(1)$ substratum, or, the electromagnetic aether, is calculated theoretically. The mass density of the $\Omega(1)$ substratum is equivalent to that 31.33195 protons contained in a box with a volume of $1.0 \mathrm{~m}^{3}$. The zero-point energy of electromagnetic fields will not appear as a source term in the Einstein's equations. Some people believed that all kinds of energies should appear as source terms in the Einstein's equations. It may be this belief that leads to the so called cosmological constant problem.
[1] S. Weinberg, Rev. Mod. Phys. 61, 1 (1989).

[2] P. J. E. Peebles and B. Ratra, Rev. Mod. Phys. 75, 2 (2003).

[3] D. Marsh, Phys. Rev. Lett. 118, 011302 (2017).

[4] C. Misner, K. Thorne, and J. Wheeler, Gravitation (Freeman, New York, 1973).

[5] P. Hamilton, M. Jaffe, P. Haslinger, Q. Simmons, H. Müller, and J. Khoury, Science 349, 849 (2015).

[6] D. Spergel, Science 347, 1100 (2015).

[7] J. Kim, P. Sikivie, and S. Weinberg, Nature 541, 464 (2017).

[8] A. Bosma, Nature 543, 179 (2017).

[9] E. Gibney, Nature 551, 153 (2017).

[10] X.-S. Wang, Progress in Physics 2, 111 (2008).

[11] X.-S. Wang, Progress in Physics 4, 25 (2008).

[12] X.-S. Wang, Physics Essays 27, 398 (2014).

[13] X.-S. Wang, Reports on Mathematical Physics 84, 35 (2019).

[14] L. Kelvin, Philosophical Magazine Series 6, 2, 161C177 (1901).

[15] M. J. Dupré and F. J. Tipler, International Journal of Modern Physics 21, 1250011 (2012).

[16] X.-S. Wang, arXiv: 1806.07724v6 [physics.gen-ph] (2018).

[17] C. Møller, The theory of relativity, second edition (Clarendon Press, Oxford, 1972).

[18] S. Weinberg, Gravitation and cosmology (John Wiley \& sons, New York, 1972).
[19] W. Rindler, Introduction to special relativity (Clarendon Press, Oxford, 1982).

[20] V. Fock, The Theory of Space Time and Gravitation, 2nd Revised Edition, Translated from the Russian by N. Kemmer (Pergamon Press, London, 1964).

[21] I. G. Currie, Fundamental Mechanics of Fluids (Cambridge University Press, 2003), 3rd ed.

[22] E. Whittaker, A History of the Theories of Aether and Electricity, from the age of Descartes to the close of the nineteenth century (Longmans, Green, and Co. London, 1910).

[23] E. Whittaker, A History of the Theories of Aether and Electricity, The Modern Theories 1900-1926 (Thomas Nelson and Sons Ltd., London, 1953).

[24] K. F. Schaffner, Nineteenth Century Aether Theories (Pergamon Press, Oxford, 1972).

[25] M. Tanabashi and et al., Phys. Rev. D 98, 030001 (2018).

[26] M. Tanabashi and et al., http://pdg.lbl.gov/2019/reviews/rpp2018-revastrophysical-constants.pdf.

[27] M. Tanabashi and et al., http://pdg.lbl.gov/2019/reviews/rpp2018-rev-physconstants.pdf.

[28] A. Borzou, Theoretical and Experimental Approaches to Dark Energy and the Cosmological Constant Problem (Springer, Switzerland, 2018). 